\title{
Re-constructing Aggregate Euro-Zone Data
}

\author{
Andreas Beyer, Jurgen A. Doornik and David F. Hendry* \\ European University Institute, Florence, \\ Nuffield College, Oxford.
}

August 7, 2000

\begin{abstract}
Reconstructing historical Euro-zone data by aggregation across individual countries is problematic because of past exchange rate changes. The approach here avoids such distortions, yet aggregates exactly when exchange rates are fixed. This is achieved by aggregating weighted withincountry growth rates to obtain euro-zone growth rates, then cumulating this Euro-zone growth rate to obtain aggregate levels. The aggregate implicit-deflator then coincides with the implicit deflator of the aggregate nominal and real data. Sub-aggregates also aggregate correctly, both regionally and temporally, as well as (e.g.) monetary sub-aggregates. The resulting Euro-zone data over the previous two decades are compared with other approaches.
\end{abstract}

\section{Introduction}

On 31 December 1998, eleven members of the European Union created a monetary union, with the Euro as its currency and monetary policy the responsibility of the European Central Bank (ECB). Because member countries previously had separate currencies susceptible to revaluations and devaluations, historical aggregate time-series data for the Euro-zone do not exist, and have to be re-created from the individual countries' records. The reasons for developing the best possible data should be clear: the ECB - and indeed any other governmental agencies - could make non-optimal policy choices by using inappropriate numbers in analyzing economic relationships that bear on its decisions. Moreover, investigators seeking to determine (say) the likelihood of the Euro-zone surviving successfully need historical data to sustain any evidence-based approach. Finally, as future data on the Euro-zone accrue, quantitative analyses will depend on having earlier time series from which to estimate and test econometric models. This paper describes appropriate ways of recreating the required data for the common currency zone when historical exchange rates in fact varied substantially - formal analyses and proofs are provided in Beyer, Doornik and Hendry (2000).

As Sir John Hicks (1939) showed, even within a given country, when relative prices change, exact aggregation is impossible - so composite goods are not well defined, and indices based on changing weighted averages of data must suffice. For example, when there are identities connecting both nominal and real magnitudes, as with national income accounting identities, the usual approach to reconciling such accounts is to deflate the sub-aggregates (such as consumption and investment), define aggregate real output as their sum, and obtain an 'implicit deflator' by dividing the nominal sum of components by that aggregate real variable. Direct aggregation of prices generally delivers a different outcome when relative prices have changed. The existence of multiple, and often conflicting, price series is especially

\footnotetext{
*Email address for correspondence: beyer@datacomm.iue.it. The second two authors' web pages are: www.nuff.ox.ac.uk/users/doornik/ and www.nuff.ox.ac.uk/economics/people/hendry.htm.
} 
problematic when inflation targeting is a primary thrust of economic policy, as in both the UK and the Euro-zone. Thus, it is important to establish a reasoned basis for selecting any given aggregation method, and the theory of index numbers has seen many important contributions in an effort to minimize distortions in our picture of 'aggregate reality': see Diewert $(1976,1988)$ for useful summaries of both the economics and statistics.

There are four main aggregation methods in common use: one could sum the levels data or the growth rates, and one could use either fixed or variable weights, in any combination. We will argue in favour of variable-weight aggregates of growth rates, namely: calculate within-country growth rates of each variable and aggregate them to a weighted-sum Euro-zone growth rate, then cumulate this Eurozone growth rate to obtain aggregate levels. Although none of the four methods can be shown to be optimal in all states of nature, nevertheless most combinations transpire to be flawed when constructing Euro-zone measures for either stock or flow variables when exchange rate shifts induce relative-price changes between individual countries in the Euro-zone. We have adopted two criteria as dominant in selecting our aggregator.

First, any method deserving serious consideration for aggregating across exchange-rate changes must at least work accurately when such changes do not occur. Since levels aggregates work well in such a state of nature, variable-weight aggregates of growth rates are most suspect. Consequently, we first consider aggregation across 'countries' which always shared a fixed exchange rate, and show that our proposed approach does provide useful aggregates, as do all the other contenders. Importantly, our proposal survives this test.

Next, it seems an essential requirement that if a variable increases (decreases) in every member state, then the aggregate should not move in the opposite direction. While this may seem a minimal requirement, we will show that levels aggregators need not perform appropriately when large currency changes occur: measured aggregates (of GDP say) can fall purely because of an exchange-rate change even when every country's GDP increases. Since fixed-weight methods deliver the same aggregates when applied to levels or changes, our proposal is left as the only one surviving both criteria. Below, we discuss the choice of weights for aggregating nominal and real GDP, prices, and money, noting that monetary weights must be used to aggregate money stocks. Finally, we also consider some of the useful properties of variable-weight growth-rate aggregates, including that sub-aggregates (e.g., regional or temporal groupings) correctly aggregate and that a unique price index is produced by the recommended weights.

The objective of this note is to explain the procedures advocated in Beyer et al. (2000) who construct Euro-zone measures for M3, nominal and real GDP, and its deflator over the two decades before the Euro was created. The created data set will be available for downloading. Section 2 evaluates the properties of aggregators that might be thought desirable, and those that are pernicious. Next, section 3 discusses aggregation under a common currency (or fixed exchange rates). Then section 4 looks at the additional problems posed by variable exchange rates. The constructed data are compared to other reconstructions in section 5. Section 6 concludes.

\section{Aggregation considerations}

Any proposed method for reconstructing 'Euroland' data should also work correctly had the member countries shared a common currency, as the USA does. Simple-sum aggregates - just adding up each member's real GDP in the common currency, say - would correctly deliver aggregate GDP under fixed rates. However, when exchange rates actually alter, seriously-misleading aggregates can ensue: consider 
the extreme case of a large devaluation by one large member, the real GDP of which appears to fall dramatically when measured in a world currency, such that aggregated real GDP falls even though real GDP rises in every member's own currency. The resulting aggregate would appear to be unrelated to say, Euro-zone unemployment, even if real GDP was the main determinant thereof within each member state. Thus, we exclude such methods from further consideration, and only examine index numbers.

Even so, either levels or growth rates could be aggregated, using either constant or variable weights which reflected the 'relative importance' of the components. Worse still, weights could be based on nominal or real variables; be the same across all variables to be aggregated or different for each series; and any date from the base year to the final might be justifiable for calculating the fixed weights - but would deliver different answers. Despite this plethora of choice, most combinations can be eliminated, as we now show.

First, fixed-weight aggregators such as Paasche or Laspeyres are unsatisfactory when relative prices change markedly, since the weights do not reflect the changed importance of the components. For example, using 1945 prices, we are 'millionaires' on average simply because of the dramatic increase in the quality and quantity of computers, since the first computer had a tiny fraction of the 'power' of a current laptop, yet cost several million dollars. Conversely, in 1999 prices, the first computer appears valueless. Thus, all fixed weight aggregators seem inadvisable for recreating 'Euroland' data given the substantial relative-price changes induced by currency devaluations and revaluations - simple sum is merely the extreme case of equal fixed weights.

By default, that leaves only variable-weight aggregators, albeit a very large class. Nevertheless, not all of these successfully avoid distortions when relative prices change sharply (as after devaluations). Since we have just established that the variables entering the index should not change sharply in such a setting, the weights have to reflect the exchange-rate shift - or the event would have no consequences! Thus, by an extension of the preceding argument about flaws in fixed-weight aggregators, this consideration precludes levels aggregators when their varying weights reflect exchange-rate changes: the aggregate can fall even though all components rise in own-currency terms. While that leaves open purchasing-power parity (PPP) weights, the difficulty of constructing those makes that an unattractive option.

Consequently, of the four initial possibilities, only growth-rate aggregators with varying weights remain. Perhaps they suffer from other drawbacks at least as serious as those used to exclude the other contenders? Section 3 argues that, despite the oddity of calculating growth rates and using variable weights when a simple sum would in fact be appropriate (as aggregating across states within a country), nevertheless the proposed method only leads to small inaccuracies, primarily due to approximating proportional changes by logarithmic differences. Thus it has no serious drawbacks - for appropriately chosen weights - as an approximation when fixed weights would work. Then, section 4 shows that only variable-weighted growth-rate aggregates remain reasonable when exchange rates are variable, leaving that as the only viable choice.

There is a burgeoning literature on creating historical Euro-zone data. Most applications of indexnumbers to date for re-creating Euroland have aggregated variables in levels, either using fixed weights (based on exchange rates, or PPP rates) for nominal and real GDP, or occasionally, fixed weights for real GDP and variable weights for nominal GDP: Winder (1997) discusses these methods, but not the approach discussed here. Some Euro-zone data are presented in ECB (1999) using fixed Euro rates (incidentally showing the concern of policy makers about this topic). Bayoumi and Kenen (1993) aggregate growth rates using fixed weights. Finally Fagan and Henry (1999) aggregate log-levels, which they show is equivalent to aggregating growth rates with fixed weights. Thus, there remains a need for developing a more justifiable data set. 


\section{Aggregation under a common currency}

For simplicity, we consider aggregating the flow data on nominal and real output for just two countries under a common currency, with the aim of also obtaining a price index, and an implicit deflator. It is easy to extend the argument to more countries, but stock variables (such as money) introduce additional issues, noted in section 3.2. The relevant growth rates are calculated using 'symmetric' log differences, rather than the more conventional choice of proportional changes. These two measures are close unless changes become large (i.e., in excess of $10 \%$ per period). Two approximations of proportional changes by log differences are needed, but are partially offsetting. We know that simple-sum aggregation must work, namely just adding the nominal outputs of the two countries to get their total nominal output etc., so we focus on whether growth-rate aggregation also works correctly. In fact, the price series resulting from our approach is unique because the aggregate price is the same as the implicit deflator. We also consider whether monetary weights - not real output weights - should be used to aggregate money stocks.

Although aggregating both nominal and real GDP in levels with constant weights (of unity) is appropriate, care is required when aggregating prices: on price indices in general, see Diewert (1976, 1988). First, the aggregate price deflator must be a variable-weighted average of the component deflators, where the correct weights are respective real GDP shares: constant weights cannot guarantee a sensible outcome since the relative sizes of the two countries can alter. Secondly, the implicit deflator obtained by dividing the nominal aggregate by the real need not coincide with the aggregate of their price indices, leading to a problem of choosing one of them to represent the 'price level' - and inflation rate - of the aggregated economy.

Our aggregator for both nominal and real GDP adds the two country's growth rates using variable weights based on their relative GDP shares in the previous period. First, note that the weights are known, and add to unity in each period. Secondly, the unlogged levels magnitudes are obtained by exponentiation then cumulating from a given first value (in practice, we matched the constructed data to the most recent official observation). Thirdly, algebraic derivations in Beyer et al. (2000) show that this cumulated changing-weight sum of growth rates will deviate from the correct aggregate primarily by rounding errors. Fourthly, we also checked the accuracy of the approach numerically for an artificial data set, and found results close to the correct aggregates. Finally, in principle, nominal GDP should be aggregated using nominal shares, but we decided to use the same real weights for both nominal and real aggregates. An important consequence, especially relevant to a world of inflation targeting, is that the resulting weighted average of the country price indices must equal the implicit deflator derived from dividing the nominal aggregate GDP by the real aggregate. Thus, any 'distortions' to the aggregate accounts affects the nominal GDP measure, which we suspect is the least relevant of the three for econometric studies and policy analyses. We conclude at this stage of the analysis that variable-weight growth-rate aggregates are a satisfactory approach under a common currency. We now note some of their useful properties.

\subsection{Sub-aggregates}

A further requirement of a usable aggregator is that it is invariant to first aggregating to sub-groups, then aggregating these. Our proposal also satisfies that property for two important classes of sub-aggregates, namely 'regional' groups and 'temporal' groups. For the regional case, four countries first aggregated in pairs to East and West, then aggregated in turn to the overall outcome, delivers the correct aggregate to the order of approximation discussed. A closely-related outcome applies for time aggregation: since the 
approach works for each frequency, correct monthly and quarterly magnitudes can be constructed. Appropriately aggregating the constructed series for stocks (end-of-period) and flows (integrals) then must match. Thus, aggregating the 'within-country' growth rates from, say, their regions delivers acceptable aggregates under constant exchange rates.

\subsection{Aggregating money stocks and money sub-aggregates}

For nominal money, however, monetary weights are needed if the outcome is to match aggregating an economy with a common currency. If alternative weights are used, such as real GDP shares as in Bayoumi and Kenen (1993), then the aggregation error becomes dependent on the different monetary growth rates of the countries involved. These growth rates can differ importantly after financial innovation in one country: if that economy has a larger share of the overall money stock than of GDP, and its monetary growth is above average because of financial innovation, then the aggregate monetary growth will be underestimated cumulatively when GDP weights are used. However, our approach continues to provide a close approximation to the actual money stock even in that setting.

Finally, sub-aggregates of money stocks also aggregate appropriately when growth-rate aggregates are used and do not depend on the order of aggregation (within countries first or money sub-aggregates first). Generalizing to countries with different exchange rates will involve the same additional approximations as for other variables discussed in section 4, assuming that the same exchange rate is used for each monetary measure.

\section{Variable exchange rates}

The main impact of variable exchange rates on the aggregation procedure proposed above is to alter the weights which enter the formulae: these will change with devaluations and revaluations since the proportions of each country in 'Euroland' GDP alter when currency values change. The weights must reflect the relative sizes of the components, and hence inherently involve both the 'scale' of each economy (measured domestically), and the exchange rates. Importantly, however, exchange rate changes do not affect the magnitudes input into the index as they do not affect the internally measured (own-country) growth rates. Still, we must confront the issue as to whether nominal or 'real' exchange rates are used in calculating weights. Because the magnitudes of growth rates are small, particularly for real growth rates, and tend to be relatively similar across countries as measured in their domestic currencies, errors in weights have a 'second-order' impact on the resulting aggregate. The effect of exchange rate shifts is, therefore, of a smaller order than when aggregating levels. In the Euro-zone, the largest member state is less than $40 \%$ of the total, and none of the larger states had very high growth rates. For example, for growth of about $1 \%$ per quarter when there is even a $10 \%$ error in calculating the dominant country's weight, then the absolute error is less than $0.07 \%$.

In 'levels' aggregation, the choice of exchange rate can matter greatly. When devaluations occur, the outcomes of any variable-weight (non-log) levels index will change sharply and suddenly, unless accurate PPP-exchange rates are used (which do not alter radically on devaluations). Different problems affect PPP-exchange rates, primarily the difficulty of constructing accurate, and uncontroversial, measures, particularly when the resulting price series may be the basis for policy models. The heavy dependence of levels aggregates on the choice of weights entails that results are a function of the particular numeraire selected, and hence vary with the frequency and magnitude of exchange-rate changes. The appropriate weights have in fact changed over time for several member states of the Euro-zone, both because of differential real growth patterns (as measured in home currency), and from currency 
changes. As noted above, when a large devaluation occurs for a given country, the imputed (common currency) value of its output falls, so measured aggregate output could actually fall even if all countries grew in terms of their domestic currencies. Thus, variable-weight levels aggregators are not viable.

Although fixed-weight aggregates are not subject to these drawbacks, the choice of weights remains crucial (see e.g., Fagan and Henry, 1999), and the method suffers from the drawbacks discussed in section 3 for common-currency aggregation. Moreover, if log-levels are aggregated, the outcome is equivalent to aggregating growth rates, as shown by Fagan and Henry (1999), so is the special case of selecting unchanging weights: should the appropriate weights alter significantly over time, the results could be misleading, and as noted above, are not correct even when there are no currency changes.

Consequently, the only approach which does not have serious drawbacks is to aggregate logdifferences with variable weights which reflect exchange-rate changes. This is the approach implemented in section 5 .

\section{Euro-zone data}

Full details of the data construction are provided in Beyer et al. (2000). Here we compare our constructed data with some published data sourced from the ECB and the OECD. Figure 1a compares the constructed $\log (\mathrm{M} 3)$ (with subscript BDH), to that from ECB (1999). The latter seems to underestimate the money stock for a prolonged period, so very different growth rates of money would be inferred at the outset and end of the discrepancy (early 1990s and around 1995 respectively). Figures 1b-c show the logarithms of nominal and real GDP respectively, with the ECB figures from the statistical archive (dated November 1999); the published OECD figures use a fixed PPP US-dollar exchange rate. For comparison, the ECB and OECD data are scaled so that the observations for 1998Q2 are identical. Different patterns of real growth emerge in panel $\mathrm{c}$ around the crucial period of 1992-3. The final panel records the price level as an index equal to unity in 1998Q2. Here the discrepancies are of policy significance, both for calculating inflation rates, and (say) real money stocks. The graphical evidence confirms the advantages of aggregating domestic growth rates with weights which reflect 'common currency' size.

However, graphs of levels are relatively 'smooth' for economic time series given the importance of trends. Consequently, figure 2 shows outcomes in differences. Panels a and b are on a common scale and show implied and constructed inflation when applying the levels method, but converting into DM (in a) and ECU (in b) respectively. These highlight the two problems of levels aggregation for inflation: implied $(\Delta p i)$ and constructed $(\Delta p)$ inflation are dramatically different, despite seasonal adjustment (panel a); and the choice of the conversion exchange rate matters greatly (comparing $\Delta p i$ across a and b). The high volatility of $\Delta p i$ is not an appetizing input to any policy decision involving inflation targeting. Panels $\mathrm{c}$ and $\mathrm{d}$ show the different outcomes for nominal and real output growth as computed by the OECD, ECB and BDH. Not only are their volatilities very different, the underlying growth rate is as well, putting a premium on appropriate data reconstructions.

\section{Conclusion}

Following the creation of the Euro-zone, new policy procedures have been set in place. To firmly base economic policy requires quantitative empirical evidence. But to quantitatively characterize 'Euroland' 

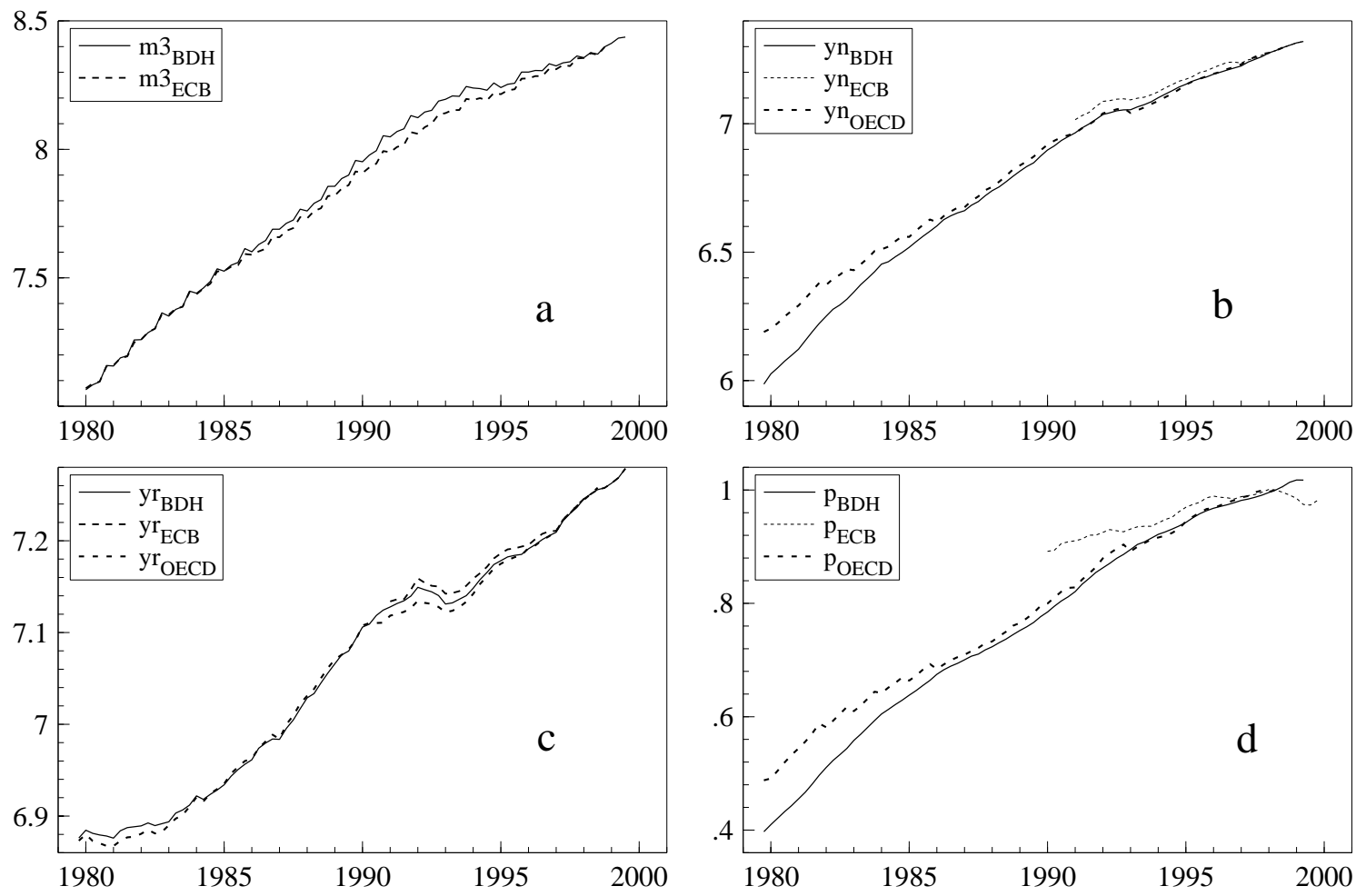

Figure 1 Comparison of constructed data with published data from ECB and OECD.
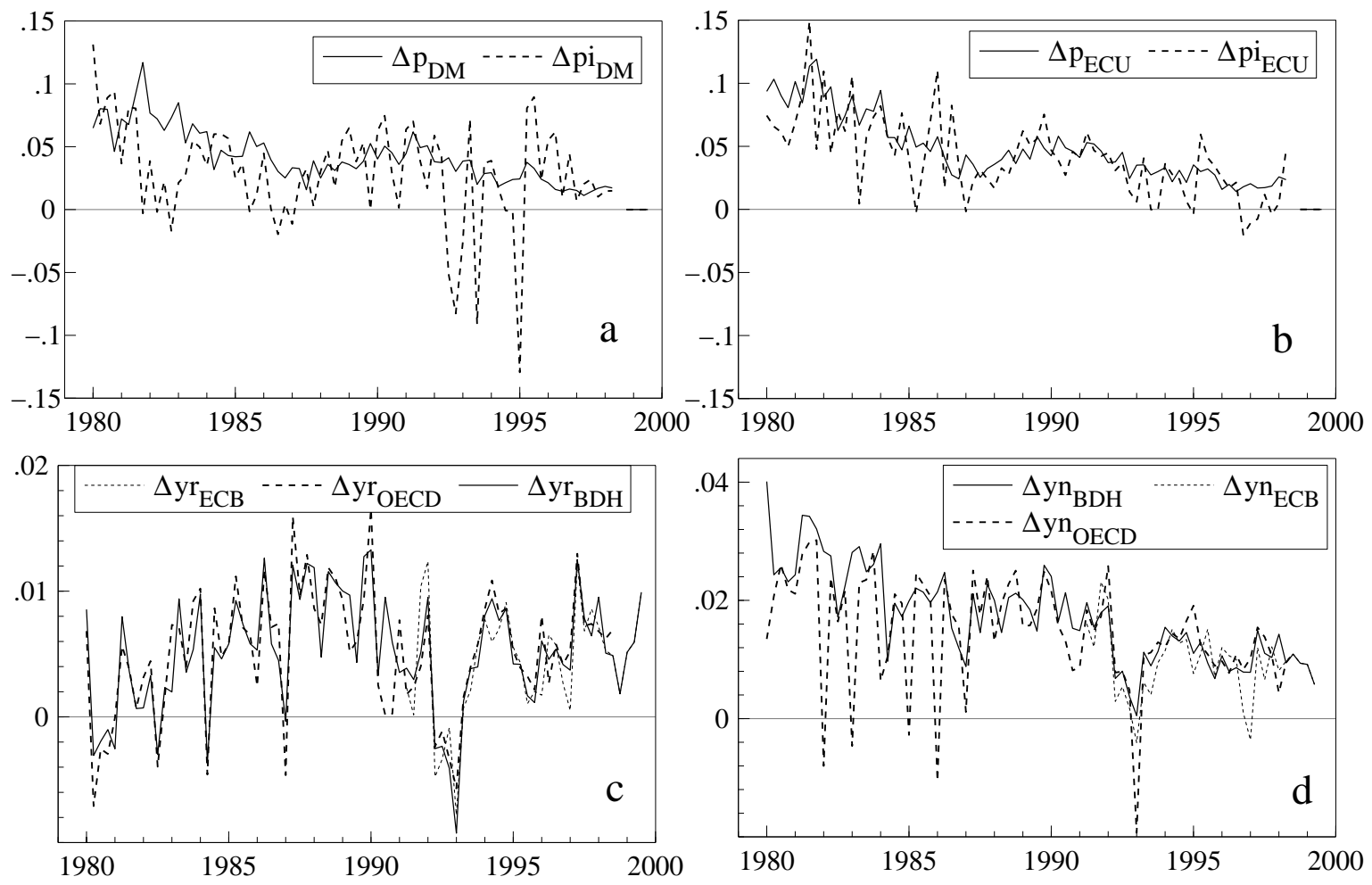

Figure 2 Comparison of constructed data with published data from ECB and OECD.

necessitates historical data thereon. Consequently, many investigators have sought to construct an historical aggregate dataset for the Euro-zone.

It is possible to construct such a dataset from the data for the member states using many different methods. However, most of these appear to suffer from important drawbacks, described above. We 
propose using a variable-weight growth-rate aggregate which was shown to avoid the main difficulties confronting either fixed weight, or levels, aggregation methods. The main problem of exchange-rate changes between member countries is less central to the aggregates resulting from growth rates, whereas that method would still aggregate almost exactly if no currency changes had occurred. Moreover, subaggregation is unproblematic, whether temporally or spatially, and the resulting implicit deflator of the nominal and real aggregates equals the aggregate implicit deflator, so a unique price measure results. This is a valuable property when economic policy seems centered on inflation targeting. We also noted that the aggregation approach was equally effective for the money stock, but suggested different weights from those used by some other investigators. Should the ECB actually use money as an 'indicator' for its decisions, then appropriate measures are important to ensure coherent econometric models thereof.

Thus, we believe the variable-weight growth-rate aggregate reconstructed dataset provides a useful basis for econometric modelling of the inputs into economic policy decisions about the Euro-zone, and intend to report our findings in due course.

\section{Acknowledgements}

Financial support from the UK Economic and Social Research Council under grant R000234954 is gratefully acknowledged. We are indebted to Iain Begg for helpful comments on an earlier draft. We also wish to thank Mike Artis, Ray Barrell, Henrik Hansen, Søren Johansen, John Muellbauer, Nigel Pain, Mark Taylor, Mike Wickens, as well as seminar participants at the EUI and Nuffield, and conference participants at 'European Monetary Union: A First Birthday Party.'

\section{References}

Bayoumi, T., and Kenen, P. B. (1993). How useful is an EC-wide monetary aggregate as an intermediate target for Europe?. Review of International Economics, 1, 209-220.

Beyer, A., Doornik, J. A., and Hendry, D. F. (2000). Constructing historical Euro-zone data. Working paper eco no.2000/10, European University Institute, Florence.

Diewert, W. E. (1976). Exact and superlative index numbers. Journal of Econometrics, 4, 115-145.

Diewert, W. E. (1988). The Early History of Price Index Research. Cambridge, MA: NBER.

ECB (1999). Euro area monetary aggregates and their role in the Eurosystems monetary policy strategy. European Central Bank Monthly Bulletin, Feb., 29-46.

Fagan, G., and Henry, J. (1999). Long run money demand in the EU: Evidence for area-wide aggregates. In Lütkepohl, H., and Wolters, J. (eds.), Money Demand in Europe, pp. 217-240. Heidelberg: Physica Verlag.

Hicks, J. R. (1939). Value and Capital. An Enquiry into Some Fundamental Principles of Economic Theory. Oxford: Clarendon Press. (2nd ed. 1950).

Winder, C. C. A. (1997). On the construction of European area-wide aggregates - A review of the issuess and empirical evidence. IFC Bulletin, 1, 15-23. 